\title{
Philosophiques
}

\section{La psychanalyse nord-américaine}

\section{François Peraldi}

Volume 4, numéro 2, octobre 1977

Philosophie et psychologie

URI : https://id.erudit.org/iderudit/203079ar

DOI : https://doi.org/10.7202/203079ar

Aller au sommaire du numéro

Éditeur(s)

Société de philosophie du Québec

ISSN

0316-2923 (imprimé)

1492-1391 (numérique)

Découvrir la revue

Citer cet article

Peraldi, F. (1977). La psychanalyse nord-américaine. Philosophiques, 4(2), 287-291. https://doi.org/10.7202/203079ar d'utilisation que vous pouvez consulter en ligne.

https://apropos.erudit.org/fr/usagers/politique-dutilisation/ 


\title{
LA PSYCHANALYSE NORD-AMÉRICAINE
}

\author{
par François Peraldi
}

La psychanalyse nord-américaine doit être critiquée, La psychanalyse nord-américaine doit être contestée, La psychanalyse nord-américaine doit être détruite.

Je parle ici de la psychanalyse telle qu'on peut l'appréhender au niveau des institutions qui ont la charge d'assurer sa transmission.

\section{Pourquoi?}

Parce que les médecins et les psychologues ont "guéri " la psychanalyse dès, qu'en la personne de Freud, elle a débarqué en Amérique du Nord.

"Ils ne se doutent pas que nous leur apportons la peste," aurait dit Freud, qui se prenait déjà pour le diable, à Jung, au moment où ils arrivaient en vue de la Statue de la Liberté.

Ce que Freud ne savait pas, c'est que les Américains avaient de bons médecins, de bons psychiatres pieux et que, si la psychanalyse était une maladie infectieuse, qu'à cela ne tienne! les bons médecins américains, avec l'aide des bons psychologues américains, prépareraient un vaccin antipsychanalytique.

Il leur fallut un peu plus de trente années pour le préparer, mais il leur suffit de la touche finale des élèves de Freud chassés d'Europe par le nazisme vers la terre d'une illusoire liberté pour qu'en pleine deuxième guerre mondiale le vaccin fut prêt, signé du triple sceau de Kris/ Hartman/ Loewenstein et béni de la main même de la fille de Freud: la bonne Anna.

Comme d'habitude, on a commencé à tester ce nouveau vaccin aux armées. Les nouveaux psychanalystes, tous bons médecins ou bons psychologues, se sont précipités, en bons patriotes, à 
l'aide des troupes américaines afin d'injecter leur vaccine psychanalytique à tous les névrosés qui leur tombaient sous la main. Leur tâche était, entre autres, de distinguer le bon déserteur (celui qui a de vrais problèmes névrotiques, qui est malade) du mauvais déserteur (celui qui s'en foutait pas mal de la nation, de l'armée et de la liberté et qui n'était pas vraiment malade). Le premier était réadapté, grâce à la vaccine psychanalytique ; son Moi, rendu plus fort grâce aux processus d'identification au Moi fort des bons analystes, lui permettait de rejoindre l'armée et le grand rêve américain ; quant au second, démasqué, il pouvait être légitimement jeté en prison puisque la vaccine n'avait pas de prise sur lui, en prison ou entre les mains des béhavioristes, ce qui revient au même.

On peut peut-être reconnaître que la plupart de ces bons psychanalytico-psychiatro-psychologo-patriotes avaient des comptes à régler avec ce nazisme que l'armée américaine s'était donné pour tâche d'écraser.

Mais comment les bons médecins et les bons psychologues ont-ils fabriqué cette vaccine psychanalytique?

De la façon dont on fabrique tout vaccin, en extirpant de la maladie elle-même le principe de sa virulence.

Ils ont donc arraché à la peste psychanalytique le principe même de sa virulence, le principe de sa révolutionnarité. À savoir ce qui fonde en son irréductible nouveauté le concept d'Inconscient - je dis le concept et non pas la notion - Je veux parler de ce que Freud a introduit dans la théorie psychanalytique sous le nom de : pulsions de mort: Todestriebe. Pulsions de mort, quelque chose qui n'a rien à voir avec ce néant qui fonde l'être, ni même avec un manque fondamental qui serait à la source de tout désir qui, de ce fait, ne serait jamais que désir de .... de ce qui manque précisément. Mais c'est quelque chose d'autre que Freud amorce lorsqu'il décrit le jeu de la bobine chez son petit-fils et qu'il nous montre comment, dans ce jeu, le langage vient à la place de ce que l'on peut tuer, faire disparaître.

On a pu entendre dire à cette table que la psychanalyse - et je parle ici de la psychanalyse promue par Freud - contestait la psychiatrie.

C'est tout aussi absurde que de dire que la conception coper- 
nicienne de l'Univers serait une contestation de la conception du Cosmos précopernicien. Une science n'en conteste pas une autre, elles appartiennent ou non à une même aire épistémologique. Au niveau d'une coupure épistémologique, il ne s'agit pas de contestation, il s'agit de révolution. Le déploiement d'une psychanalyse, telle qu'amorcée par Freud à partir de la coupure épistémologique instaurée par l'introduction de la pulsion de mort dans la théorie, implique l'effacement, la disparition, la destruction de la psychiatrie tout autant d'ailleurs que de la psychologie.

La mise en place de la pulsion de mort au principe même de l'Inconscient introduit dans la notion de sujet le principe d'une division irréductible et qui n'en finit plus de se répéter dans le sens d'une pluralisation infinie du sujet. Cette division n'a d'ailleurs rien à voir avec les oppositions binaires dissymétriques du type santé/maladie, normalité/folie, maître/esclave. Le processus introduit par Freud commence par le "Je est un Autre" de Rimbaud, il se continue par le "Je suis mon père, je suis ma mère, je suis le roi, etc ... d'Artaud pour s'épanouir dans la parole du diable: "Je suis légion, " si justement rapportée hier par Roger Savoie.

Avec l'introduction de la pulsion de mort, Freud coupait avec toute cette aire épistémologique où les sciences dites de l'homme sont centrées par le cogito cartésien autour duquel tournent des choses comme, - je cite en vrac: la pensée, le savoir, la conscience, le Moi, la vision continuiste de l'histoire, le progrès, la guérison, etc...

Avec l'introduction de la pulsion de mort, Freud introduisait une conception plurielle du sujet. Contre le "Je suis UN ", le " Je suis légion" ou le "Je suis personne". Autrement dit, il introduisait la destruction de la notion même de sujet, donc évidemment d'objet.

Mais les bons médecins américains, les bons psychologues, la bonne Anna ne pouvaient pas laisser passer une chose pareille, puisque la mort est précisément ce qui disqualifie la médecine; et puis, comment être bon quand on ne peut plus dire "je ", et que l'on se réduit à n'être que bon à rien? D'un petit tour de passepasse, un simple faux-sens, il suffisait de traduire Todestriebe (pulsions de mort) par death instincts (instincts de mort) - puis- 
qu'il est bien connu qu'on ne lit plus Freud en allemand mais en anglais - pour renvoyer le tout bien empaqueté chez les biologistes et leur dire : occupez-vous en, nous on s'occupe des pulsions, pas des instincts. La pulsion agressive, oui ! ça se sublime dans la guerre, par exemple!

Un inconscient, que la pulsion de mort ne centre plus, devient parlable, la mort, elle, ne se parle pas.

Un inconscient, que la pulsion de mort ne centre plus, devient interprétable.

Ce n'est même plus un inconscient, c'est un oubli, c'est du passé, et le passé une fois remémoré, le temps perdu retrouvé, grâce aux bons analystes qui savent interpréter vos rêves et vous rendre votre passé que la maladie avait effacé, - une fois ce temps perdu retrouvé, il n'y a plus d'inconscient. Vous êtes $U N$, vous êtes une personne totale, un bon soldat, par exemple, vous êtes devenu un Moi, maître de lui comme de l'univers, en un mot vous êtes unifié dans le registre de l'Imaginaire à tout jamais coupé du Réel, qui est précisément le lieu du meurtre, et possédé par le langage qui devient pour vous le réel tout autant que la réalité.

L'imaginaire, c'est précisément ce lieu où l'on peut vous faire prendre des vessies pour des lanternes, les causes de vos oublis pour les symptômes d'une hystérie ou d'une névrose, car c'est le lieu du savoir psychiatrique ou psychologique, mais aussi le lieu où l'on peut vous faire prendre la guerre au Tiers-Monde pour le plus cher de vos désirs, car c'est le lieu de l'aliénation.

\section{Comment détruire la psychanalyse nord-américaine?}

On peut faire appel ici à des notions deleuziennes. La psychanalyse nord-américaine a été territorialisée par les médecins et les psychologues, elle a été rattachée aux appareils de pouvoir médicaux et aux institutions médicales.

La détruire ne veut dire rien d'autre que la déconstruire: défaire ce qui la rattache aux appareils de pouvoir médicaux.

La déterritorialiser : l'arracher aux mains des médecins et des psychologues.

La pluraliser : la distribuer à tout le monde. Tout le monde peut devenir psychanalyste. 
Il faut multiplier les branchements de la psychanalyse avec les autres domaines révolutionnaires, lui restituer sa violence épidémique de peste, lui restituer ce qui la fonde, au-delà de la coupure épistémologique instaurée par Freud, comme science révolution. naire : le concept de pulsion de mort.

C'est au fond par la violence fasciste, inhérente à tout mouvement révolutionnaire, que la psychanalyse peut reprendre sa place au coeur des pratiques révolutionnaires et contre les tenants de la contre-révolution et du bourgeoisisme : les médecins, les psychiatres et les psychologues en tant qu'agents des appareils de pouvoir médicaux. Ceux que l'on peut désigner d'un terme de Paul Nizan qui trouve ici toute sa violence : les chiens de garde.

École de Traduction, Université de Montréal. 